\title{
CHILDHOOD PSYCHOPATHOLOGY IN CHILDREN OF WOMEN WITH EATING DISORDERS.
}

\section{FINDINGS FROM DANISH NATIONAL BIRTH COHORT}

Manuela Barona, Institute of Child Health, UCL, London, UK

Anne Marie Nybo Andersen, University of Copenhagen, Denmark

Dr Nadia Micali, Institute of Child Health, UCL, London, UK; Department of Psychiatry, Icahn Medical School at Mount Sinai, New York, NY, US; Mindich Child Health and Development Institute, Icahn Medical School at Mount Sinai, New York, NY, US

Running title: Psychopathology in children at risk for ED

Correspondence: Manuela Barona, MSc., $4^{\text {th }}$ Floor Institute of Child Health, 30 Guilford Road, WC1N 1EH, London, UK; 02079052166, m.martinez-barona@ucl.ac.uk 


\section{ABSTRACT}

Objective: We aimed to investigate the effect of maternal eating disorders (ED) on childhood psychopathology, early delays in cognitive, motor and language development; mother and child relationship, and child temperament in a community-based cohort: the Danish National Birth Cohort (DNBC).

Method: Data were obtained prospectively on 48,403 children at 18 months and 46,156 children at 7 years. Data on cognitive, motor and language development, temperament and attachment were obtained at 18 months; data on child psychopathology were obtained at 7 years of age, using the Strengths and Difficulties Questionnaire (SDQ). Children of mothers with lifetime diagnosis of anorexia nervosa (AN, n=931), lifetime diagnosis of bulimia nervosa $(\mathrm{BN}, \mathrm{n}=906)$ and both $(\mathrm{AN} \& \mathrm{BN}=360)$ were compared to children of mothers without an ED ( $n=46,206)$.

Results: Girls of women with lifetime AN had higher odds of having emotional problems, and girls of women with lifetime $\mathrm{BN}$ of having conduct problems compared with children of healthy women. Boys of women with lifetime AN had higher odds of total, emotional and conduct problems; boys of women with lifetime BN had higher odds of total, conduct, hyperactivity and peer difficulties compared to children of women without an ED. Boys of women with lifetime $\mathrm{AN}$ and $\mathrm{BN}$ had higher odds of total, emotional and peer problems compared to children of healthy women.

Conclusion: Maternal ED are associated with childhood psychopathology in both boys and girls. Boys seemed at higher risk for psychopathology in this sample. Associations between emotional disorders across genders in children of mothers with lifetime AN, and hyperactivity and peer difficulties in boys of mothers with lifetime $\mathrm{BN}$ confirm and extend previous findings and point to possible shared risk between ED and other psychopathology.

Keywords: DNBC, eating disorders, psychopathology, SDQ, temperament, children

\section{SIGNIFICANT OUTCOMES}


- Children of women with ED are at increased risk of psychopathology across several domains.

- The links between maternal BN and childhood hyperactivity and conduct problems, and maternal AN and childhood emotional problems might underlie shared genetic risk, however, this requires further research.

- Studying children at risk for eating disorders helps clarify possible intermediate phenotypes for the disorder as well as shared vulnerabilities with other disorders.

\section{LIMIATIONS}

- Maternal eating disorder status was obtained using self-report during interviews.

- The data on the children were obtained from mothers (shared method variance).

- We found evidence of selective attrition amongst women with ED, therefore generalizability of this study might be affected. 


\section{INTRODUCTION}

The effect of maternal psychiatric disorders on their offspring's mental health is well documented $(1,2)$. It has been established that children of parents with psychiatric disorders are at a higher risk of developing a range of emotional, behavioural, cognitive and social difficulties(3). In particular, recent large-population based studies have highlighted an increase in the prevalence of mental health disorders in children with parental history of a psychiatric disorder $(4,5)$. This may be both due to genetic factors and factors associated with the environment(6). Despite this evidence the effect of maternal eating disorders (ED) on their offspring general psychopathology has been less studied.

The large majority of research on the effect of maternal eating disorders on children has focused on the early years of child development, when formal psychopathology is rare(7). Case series have reported psychological disturbance in areas independent of eating in the offspring of mothers with ED, such as emotional, $\operatorname{speech}(8$, 9), behavioural problems(10) and general psychopathology(11). However, these findings rely on small clinical samples and therefore might be biased. Two recent studies $(12,13)$ using data from a large population-based study, the Avon Longitudinal Study of Parents And Children (ALSPAC) investigated the effect of maternal eating disorders on childhood psychopathology at three and a half years(12) and in late childhood and early adolescence(13). Children of women with eating disorders, both anorexia and bulimia, had a higher risk of developing psychopathology in infancy (at 3 and 1/2 years) than unexposed children. These children also had higher prevalence of psychiatric disorders in late childhood (age 10 years) (13). Recent research has also found that children of mothers with ED show differences in cognitive performance compared to healthy children, such as difficulties with social understanding, visual-motor planning and abstract reasoning(14). However, less is known about early childhood (ages 5 to 7), when children start formal education, leading to new demands on cognitive and social functioning and potential difficulties becoming more prominent.

Another main focus in previous research has been the effect of maternal eating disorders on mother child bond and parenting(15), because of the effect that ED have on everyday functioning. Parental eating disorders are likely to adversely affect one's ability to care for a child(16) and studies have indeed shown that that eatingbased psychopathology is related to parenting difficulties. Studies on mother-child relationship have mostly 
focused on interactions during meal times in small clinical samples(17). However, not much attention has been given to understanding mother child relationship irrespective of eating situations (which can cause a lot of distress for mothers). In fact, research has shown that mothers with ED report that their ED symptomatology interferes with their general parenting practice (18). Therefore clarifying the associations between maternal ED and parenting can help develop adequate intervention and prevention strategies.

Aims of the study:

Based on the gaps in the literature identified above and the need for replication of results (19), we aimed to investigate the effect of maternal eating disorders on children's psychopathology as measured by the Strengths and Difficulties Questionnaire at 7 years of age, which measures levels of conduct, emotional, hyperactivity, social problems and overall psychopathology (although not eating disorder psychopathology). We also studied whether maternal eating disorders predicted early child development: motor, cognitive and language; as well as mother child difficulties and child difficult temperament at 18 months. Based on previous findings on gender differences in past studies in children of mothers with ED we focused on specific differences stratified by gender. We also aimed to explore whether child temperament might be a mediator in the associations between maternal ED and child psychopathology

\section{METHODS}

\section{Participants}

The Danish National Birth Cohort (DNBC) is a longitudinal population study established to explore foetal growth, early life and its determinants(20). Between 1996 and 2002, 101,042 women consented to take part in the study; 92,892 pregnant women were eligible after participating in the initial telephone interview at 12-16 weeks gestation. Women with multiple pregnancies were included. Information was collected using computerised telephone interviews, which occurred at four different time-points: at 12 and 30 weeks gestation and at 6 and 18 months postnatal and a follow up at 7 years old(21). For more information on the cohort, refer to www.DNBC.dk. 
Data on the main predictor (maternal ED) was obtained by interview (around 12 week gestation) $(\mathrm{N}=83,820$ ). Women were asked whether they had ever suffered from anorexia or bulimia nervosa (BN)(22). These women were grouped into the following exposure groups for lifetime (at any point in their life) disorder: lifetime bulimia nervosa $(\mathrm{BN})(\mathrm{N}=1,751,2.1 \%)$ and lifetime anorexia nervosa $(\mathrm{AN})(\mathrm{N}=1,673,2.0 \%)$. Women who answered yes to having ever suffered from both disorders were included as a separate exposure group $(\mathrm{N}=658,0.8 \%)$, given previous evidence from Micali et al. 2007(23) that this group differs from the other two. Women with no history of ED were classified as unexposed ( $\mathrm{N}=79,738,95.1 \%)$.

\section{Outcomes (18-months)}

\section{Developmental milestones}

In the 18-month interview, mothers answered 11 questions pertaining to delays in their infant's milestones. Five questions pertaining to motor development delays, (two assessed age for sitting and standing up, and three assessed whether the infant could climb stairs, remove his/her socks and shoes, and drink from a cup or glass by him-/herself). Six cognitive and language development questions were also asked (25). A developmental delay summary score for motor ( $0-5$ points) and cognitive/language ( $0-6$ points) development was calculated. Consistent with previous studies (24), to summarize motor, cognitive and language milestones, we defined as "delayed" those infants whose scores for motor or cognitive/language development corresponded to the highest $5 \%$ of all infants for each of the 2 summary measures (motor delay and cognitive/language delay). This cut-offs have been used previously in the DNBC cohort (24).

\section{Child temperament}

Mothers were asked to indicate the frequency of a set of child characteristics. Whether the child was restless in his/her sleep, whether he/she was more or less active than other children, whether he/she was a happy child, and whether he/she was cautious and guarded. A summary score was obtained by adding the responses to three set of questions. Higher scores indicated a more difficult temperament. The scale was not normally distributed and could not be normalized therefore scores were dichotomised using the top $10 \%$ as indicating difficult temperament. The dichotomised variable was used in all analyses. 


\section{Looking after the child}

Mothers were asked one question on how difficult it had been to take care of the child since he/she was born, when the study children were 18 months old. The variable was coded as categorical, $(1=$ very easy, $2=$ fairly easy, $3=$ difficult, $4=$ very difficult). Higher scores in the variable are associated with more difficulties.

\section{Outcomes (at 7 years of age)}

\section{Psychopathology}

A total of 57,282 (63\% of those originally enrolled) women completed the SDQ(25) when the child was 7 years of age. The SDQ is a brief 25-item questionnaire, validated to assess psychopathology in children and adolescents(25); it has been validated in children across four large Danish cohorts, supporting its use in the Danish population [reliability (chronbach's alpha: 0.75-0.88)] (26). The reliability and validity of the SDQ makes it a useful short measure of psychopathology in children and adolescents (27) and all scales are associated with the relevant DSM-IV diagnoses (27). Furthermore, the SDQ algorithm capitalises on the high level of detection of comorbidities; a well-recognised feature of child psychopathology (28). The SDQ is completed by parents about their child's behaviour in the past six months and comprises five different subscales (emotional, conduct, hyperactivity/inattention, pro-social and peer-relations) as well as a total scale. The responses for each item are coded in a 3-point Likert scale (not true, somewhat true, certainly true). We used recognised cut-offs for the SDQ (which roughly correspond to $80 \%$ of children: "normal" range, $10 \%$ : borderline range and 10\%: clinical range), commonly used in clinical practice. The specific cut-off scores used were developed by Niclasen in 2012 based on a sample of almost 60,000 Danish children and are used to identify the $20 \%$ of the sample with the highest problem scores (http://www.sdqinfo.org/DanishNorms/DanishNorms.html)(29). The categories normal, borderline and clinical so derived were used as an ordered variable.

\section{Attrition and missingness}

Women were eligible for the study if they had answered questions about their lifetime ED and had a live born child $(\mathrm{N}=83,820)$. We only included the first child amongst women who had more than one child in the 
cohort-to avoid non-independence of observations. Data on 48,403 children and mothers were available for the analyses investigating motor, cognitive and language development, temperament and attachment in children of mothers with ED and non-ED. Data on 46,156 children and mothers were available for the analyses investigating childhood psychopathology in children of mothers with ED and non-ED.

Patterns of missingness and attrition in the sample were investigated using logistic regression prior to any analyses, using missingness as the outcome variable and socio-demographic variables (maternal age, maternal employment status, co-habituating status, and parity) and maternal ED as predictors. Missingness on the outcomes under study was associated with maternal ED. Missingness on data at 18 months was also associated with higher parity, higher maternal age and lower maternal social status.

\section{Covariates}

Data on maternal age, maternal employment status, parity and birth-weight were obtained from the Danish National Patient Discharge Register and Central Person Register and were included as covariates in adjusted analyses.

\section{Data analysis}

All continuous variables were examined individually to check for inconsistencies/outliers and normality using tabulations and histograms. Given the large sample (over 1000), statistically significant deviations from normality shown in common tests can be irrelevant, therefore the data was checked for normality initially using normality tests (Kolmogorov-Smirnov Test) and then using histograms (with a reference normal distribution curve). No inconsistencies or outliers where found in the final sample. The primary outcome variables investigated in the paper were the following: child development, temperament, looking after the child, and SDQ. Pearson or spearman correlations, depending on the distribution of the variables, were used to investigate correlations between outcome variables. The distribution of the covariates was studied according to the main predictor using chi-square, for categorical variables, and F-tests, for continuous variables, in order to provide a comparison between exposure groups. 
Associations between maternal ED and SDQ scores were investigated using ordinal regression analyses, in which the OR represent the increase in the ordered odds of being in the abnormal (clinical), vs borderline, vs normal range for each subscale (and the total score). Associations between maternal ED and the outcomes at 18 months were investigated using logistic regression. All assumptions for ordinal regression analyses were met, including the proportional odds assumption, which was examined using the parallel lines test in SPSS.

All analyses were stratified by gender based on previous results highlighting differences based on gender. Initially crude analyses were run. Additional a priori confounders (maternal age, maternal employment status, birth-weight and parity) were included in adjusted models. All analyses were run using SPSS 21(SPSS Inc., USA), and a two-tailed significance level of $5 \%$ was used.

\section{Post-hoc mediation analyses}

We investigated the role of temperament as a possible mediator of the associations between maternal ED and child psychopathology. Mediation was tested in SPSS following the methodology by MacKinnon and Dwyer for mediation with categorical variables(30).

\section{Ethical approval}

The DNBC was approved by the Committee on Biomedical Research Ethics and all women who participated in the study gave informed written consent. The present study was approved by the DNBC steering committee (ref. 2010-33).

\section{RESULTS}

\section{Socio-demographic data}

Results are shown in table 1. Socio demographic characteristics were studied across exposed and unexposed women. Significant differences were found for education, parity, employment status, birth weight and maternal age.

Maternal ED and development, temperament, and mother child relation at 18 months of age 


\section{Development}

Girls: Girls of mothers with lifetime ED were comparable to girls of non-ED women (table 2).

Boys: Boys of women with lifetime AN were less likely to have delayed cognitive and language development in crude and adjusted analyses $(\mathrm{OR}=0.75,95 \% \mathrm{CI}$ : 0.62-0.91) compared to boys of unexposed women (Table 2).

\section{Child temperament and mother-child relation}

Girls: Women with lifetime BN were more likely to perceive their daughter as having a difficult temperament in crude and adjusted analyses ( $\mathrm{OR}=1.85,95 \% \mathrm{CI}$ : 1.30-2.65) compared to unexposed women (Table 2).

Women with lifetime ED were more likely to report difficulties looking after their daughter in crude and adjusted analyses (respectively $\mathrm{BN}$ : $\mathrm{OR}=2.07,95 \% \mathrm{CI}: 1.42-3.03$; $\mathrm{AN}$ : $\mathrm{OR}=1.69,95 \% \mathrm{CI}: 1.12-2.55$; $\mathrm{AN} \&$ BN: OR=1.96, 95\%CI: 1.02-3.74) compared to unexposed women (Table 2).

Boys: Women with lifetime AN and those with both lifetime AN and BN were more likely to perceive their son as having a difficult temperament in crude and adjusted analyses ( $\mathrm{OR}=2.33,95 \% \mathrm{CI}$ : 1.34-4.07) compared to unexposed women. (Table 2).

Women with lifetime BN were more likely to report difficulties looking after their son in crude and adjusted analyses (OR=1.71, 95\% CI: 1.21-2.41) compared to unexposed women (Table 2).

\section{Maternal ED and child SDQ at 7 years}

Girls: Girls of women with lifetime BN had higher odds of conduct difficulties in crude and adjusted analyses $(\mathrm{OR}=1.41,95 \%$ CI: 1.09-1.83) compared to girls of unexposed women (Table 3). Girls of women with lifetime AN had higher odds of emotional difficulties in crude and adjusted analyses (OR=1.28,95\% CI: $1.00-1.63)$ compared to girls of unexposed women (table 3). 
Boys: Boys of women with lifetime BN had higher odds of having total, conduct, hyperactivity and peer difficulties in crude and adjusted analyses (respectively: $\mathrm{OR}=1.51,95 \% \mathrm{CI}: 1.23-1.84 ; \mathrm{OR}=1.32,95 \% \mathrm{CI}$ : 1.05-1.66; OR=1.33, 95\%CI: $1.08-1.64 ; \mathrm{OR}=1.37,95 \% \mathrm{CI}: 1.12-1.67)$ when compared to boys of unexposed women (Table 3). Boys of women with lifetime AN had higher odds of total, emotional and conduct difficulties in crude and adjusted analyses (respectively: OR=1.37, 95\% CI: $1.10-1.70$; OR=1.56, 95\%CI: 1.23-1.98; OR=1.32, 95\% CI: 1.04-1.67) compared to boys of unexposed women (Table 3).

Boys of women with lifetime AN and BN had higher odds of total, emotional and peer difficulties in crude and adjusted analyses (respectively: $\mathrm{OR}=1.91,95 \% \mathrm{CI}: 1.41-2.59$; OR $=1.92,95 \% \mathrm{CI}: 1.36-2.70 ; \mathrm{OR}=1.71$, 95\% CI: 1.26-2.32) compared to boys of unexposed women (table 3).

\section{Post-hoc mediation analyses}

There was a significant indirect effect of maternal lifetime BN on SDQ total, emotion, conduct, hyperactivity and peer scores mediated via child temperament (respectively: estimate $=0.16, \mathrm{SE}=0.01, \mathrm{p}<0.01$; estimate $=0.24, \mathrm{SE}=0.01, \mathrm{p}<0.01$; estimate $=0.08, \mathrm{SE}=0.01, \mathrm{p}<0.01$; estimate $=0.07, \mathrm{SE}=0.01 \mathrm{p}<0.01$ and estimate $=0.10, \mathrm{SE}=0.01, \mathrm{p}<0.01)$. The direct effects from maternal lifetime $\mathrm{BN}$ to SDQ total, conduct and hyperactivity scores were also significant (respectively: estimate $=0.05, \mathrm{SE}=0.01, \mathrm{p}<0.01$; estimate $=0.29$ $\mathrm{SE}=0.10, \mathrm{p}<0.01$ and estimate $=0.32, \mathrm{SE}=0.09, \mathrm{p}<0.01)$.

Child temperament also mediated the effect of maternal lifetime AN on SDQ total, emotion, conduct, hyperactivity and peer scores (indirect effects respectively: estimate $=0.16, \mathrm{SE}=0.01, \mathrm{p}<0.01$; estimate $=0.24$, $\mathrm{SE}=0.01 \mathrm{p}<0.01$; estimate=0.08, $\mathrm{SE}=0.01, \mathrm{p}<0.01$; estimate $=0.07, \mathrm{SE}=0.01, \mathrm{p}<0.01$ and estimate $=0.10$, $\mathrm{SE}=0.01, \mathrm{p}<0.01)$. There were no significant direct effects.

\section{DISCUSSION}

A recent population-based prospective study showed that maternal ED were associated with childhood psychopathology outcomes, particularly emotional disorders in early adolescence in both boys and girls. However attrition in early adolescence means small sample sizes were achieved by this stage (31). In the present study, we aimed to extend and replicate these findings by using data from a larger population-based 
study and investigate the development of psychopathology at seven years of age. Reproducibility is a defining feature of science and replication of results using different samples in different countries, with a different confounding structure can lead to increased confidence in the findings(19). We found that children of women with eating disorders were at risk of developing psychopathology in early-childhood across most domains (emotional, conduct, hyperactivity and peer difficulties); and were more likely to have a difficult temperament. We also found that women with ED were more likely to report their relationship with their child as being difficult. Boys of mothers with AN were less likely to have a delayed language and cognitive development. Mediation analyses showed that there was a significant indirect effect of maternal AN and maternal $\mathrm{BN}$ on psychopathology mediated via child temperament at 18 months.

Boys of women with $\mathrm{BN}$ had higher odds of developing psychopathology across most domains. In particular, findings that hyperactivity in boys was associated with maternal $\mathrm{BN}$ are consistent with our previous study (12). One possibility for this, is that $\mathrm{BN}$ and attention deficit and hyperactivity (ADHD) disorder share several key features. Studies have shown that individuals with a diagnosis of ADHD are at greater risk of developing an $\mathrm{ED}(32-34)$ and this association is particularly true for $\mathrm{BN} /$ binge eating(35). However, in our study, this was only significant for boys. A possible explanation for this finding could be due to the gender distribution of hyperactivity and attention difficulties, which have been found to be more common in boys. An alternative explanation is that mothers might be more likely to report these difficulties selectively by gender. Despite possible explanations for the selectivity of results, the findings partly underscore the well-replicated association between hyperactivity/inattention and $\mathrm{BN}(36,37)$.

Both girls and boys of women with AN had higher odds of having emotional problems. These results are consistent with previous research which highlighting the association between AN and emotional/anxiety disorders $(38,39)$, and are consistent with our results from the ALSPAC study in different age groups(12, 31). Significant comorbidity for major depression in family probands and monozygotic twins has been found in past studies, supporting the hypothesis of a shared genetic transmission between ED and anxiety disorders. However, as with the association between hyperactivity/inattention, further research is needed in order to discern if the association is due to shared genes or might indicate an intermediate phenotype for AN. 
Another interesting finding relates to peer difficulties in this sample. Results showed that boys of women with a lifetime history of $\mathrm{BN}$ (both those who reported $\mathrm{BN}$ only and $\mathrm{AN}+\mathrm{BN}$ ) have more peer problems when compared to children of healthy women. The questions in the SDQ that load to the peer problems reflect problems engaging and communicating with other children, which could be a reflection of social communication problems. We have previously shown that children at risk for $\mathrm{BN}$ had difficulties in recognising emotions and had worse social communication(40) compared to controls. These results might point to a possible overlap between $\mathrm{BN}$ and social communication difficulties.

Although the literature on maternal ED and mother-child relationship is scant, the results observed in this study replicate and extend past research. An early study(41), found that the conflict between the demands of motherhood and those of the ED created tension in the relation between mother and child. We also found that mothers with ED tended to report their children as having a more difficult temperament. However, because the present study used maternal report rather than direct observational measures for mother child relation and child temperament, the nature of the associations remains unclear. One possible explanation is that children of mothers with ED are objectively more difficult than those of healthy control mothers, however, we cannot discard the possibility that mothers with ED may perceive their child as being more difficult. However, even if these difficulties are in 'the eye of the beholder', mothers perception is likely to impact on their own sense of being a mother (42) and are likely to negatively impact on the child's later development.

We found that boys of women with AN had lower odds of having delayed cognitive and language development. A recent meta-analysis found that patients with AN score higher on the National Adult Reading Test and the Wechsler Intelligence Scales(43). These results are consistent with previous findings from our study in children of mothers with $\mathrm{AN}(14)$, however, these were not stratified by gender and therefore we do not know if this was true for both girls and boys. Further clarification is needed to understand gender differences in the neuropsychological profile of children at high-risk for ED. In this study, we did not replicate the association between maternal eating disorder and motor development. This might be due to the latter study using an objective measure of development, rather than the maternally reported one used in the current study. 
Our mediation analyses suggest some differences in mediation pathways across maternal diagnosis; for example, whilst child temperament partially mediated the effect of $\mathrm{BN}$ on child psychopathology (pointing to a potential shared genetic and environmental effect), for maternal AN, there was no direct effect of the ED on child psychopathology, the relationship was fully mediated by child temperament. These results should be examined with caution as all measures are obtained via maternal report and could therefore be biased. Important next steps needed are for research to test theories of possible disorder specific pathways to risk, including accounting for possible child moderators.

\section{Strengths and limitations}

The main strengths of this study are the large sample size, the prospective data collection, and the availability of register-based information on socio-demographic data. This is the largest study investigating maternal ED and the effect of childhood psychopathology to date.

Limitations have to be taken into account: firstly the information on maternal ED was obtained from selfreport during interviews. However, a recent study validated self-reported ED in a similar population based sample of pregnant women with very good sensitivity and specificity of self-reported AN and BN(44). Furthermore, there is evidence from past research that self-report measures might be as reliable, as other, more frequently, used measures(45). Another important limitation is that data on the children were obtained from mothers (shared method variance). The variables on child temperament and difficulties looking after the child were based on questionnaires designed for DNBC and have therefore not been validated. We found evidence of selective attrition amongst women with ED, therefore generalizability of this study might be affected. It is difficult to know whether the selective attrition might be reflective of more severely ill mothers being lost to follow up or whether children with lower levels of psychopathology might be lost to follow up, therefore whether results are biased towards the null or not. However in a previous similar study(13), children with higher psychopathology were more likely to be lost to follow-up therefore suggesting that OR might be biased towards the null. This means that we may be underestimating the effect found in our study if we expect more severely ill mothers to be lost to the study. The present study had several comparison, however, there were 
only four primary outcomes and three groups that are compared to a reference group, therefore less than one result would be due to chance.

Concluding remark:

This study has important research implications for our understanding of shared diathesis for psychopathology and ED, however there are limitations to the results, the main one being that all measures were obtained via maternal reports. Further studies are needed to better understand the shared diathesis for psychopathology and ED. Importantly, these studies should include more objective measures of psychopathology. At risk studies have the potential to provide important information about intermediate phenotypes. Further investigation of specific risk pathways and mechanisms in the intergenerational transmission of psychopathology in children of parents with ED is needed. Studies focusing on children at risk and on understanding risk mechanisms will be indispensable for developing early intervention programs as well as prevention strategies, and to improve our understanding of ED and their pathophysiology.

\section{ACKNOWLEDGMENTS}

We are extremely grateful to all the families who took part in this study and the whole DNBC team. The Danish National Research Foundation established the Danish Epidemiology Science Centre that initiated and created the Danish National Birth Cohort. The cohort is a result of a major grant from this Foundation. Additional support for the Danish National Birth Cohort is obtained from the Pharmacy Foundation, the Egmont Foundation, the March of Dimes Birth Defects Foundation, the Augustinus Foundation, and the Health Foundation. This publication is the work of the authors and they will serve as guarantors for the contents of this paper. This study was funded by an International exchange grant to Dr Micali and Prof NyboAndersen from the Royal Society, UK.

\section{DECLARATION OF INTEREST}

No financial or non-financial conflicts of interest exist for any of the authors.

\section{REFERENCES}


1. Goodman SH, Gotlib IH. Risk for psychopathology in the children of depressed mothers: a developmental model for understanding mechanisms of transmission. Psychol Rev. 1999;106(3):458-90.

2. Satyanarayana VA, Lukose A, Srinivasan K. Maternal mental health in pregnancy and child behavior. Indian Journal of Psychiatry. 2011;53(4):351-61.

3. Leverton TJ. Parental psychiatric illness: the implications for children. Current Opinion in Psychiatry. 2003;16(4):395-402.

4. Dean K, Stevens H, Mortensen PB, Murray RM, Walsh E, Pedersen CB. Full spectrum of psychiatric outcomes among offspring with parental history of mental disorder. Archives of general psychiatry. 2010;67(8):822-9.

5. McLaughlin KA, Gadermann AM, Hwang I, Sampson NA, Al-Hamzawi A, Andrade LH, et al. Parent psychopathology and offspring mental disorders: results from the WHO World Mental Health Surveys. The British Journal of Psychiatry. 2012;200(4):290-9.

6. Rutter M. Psychiatric disorder in parents as a risk factor for children. Prevention of mental disorders, alcohol and other drug use in children and adolescents. 1989:157-89.

7. Brinch $\mathrm{M}$, Isager $\mathrm{T}$, Tolstrup K. Anorexia nervosa and motherhood: reproduction pattern and mothering behavior of 50 women. Acta Psychiatrica Scandinavica. 1988;77(5):611-7.

8. Hodes M, Timimi S, Robinson P. Children of mothers with eating disorders: a preliminary study. European Eating Disorders Review. 1997;5(1):11-24.

9. Franzen $U$, Gerlinghoff M. Parenting by patients with eating disorders: Experiences with a motherchild group. Eating Disorders. 1997;5(1):5-14.

10. Timimi S, Robinson P. Disturbances in children of patients with eating disorders. European Eating Disorders Review. 1996;4(3):183-8.

11. Stein A, Woolley H, Cooper S, Winterbottom J, Fairburn CG, Cortina-Borja M. Eating habits and attitudes among 10-year-old children of mothers with eating disorders: longitudinal study. Br J Psychiatry. 2006;189:324-9.

12. Micali N, Stahl D, Treasure J, Simonoff E. Childhood psychopathology in children of women with eating disorders: understanding risk mechanisms. J Child Psychol Psychiatry. 2013.

13. Micali N, Stavola B, Ploubidis GB, Simonoff E, Treasure J. The effects of maternal eating disorders on offspring childhood and early adolescent psychiatric disorders. International Journal of Eating Disorders. 2013.

14. Kothari R, Solmi F, Treasure J, Micali N. The neuropsychological profile of children at high risk of developing an eating disorder. Psychol Med. 2013;43(7):1543-54.

15. Woodside DB, Shekter-Wolfson LF. Parenting by patients with anorexia nervosa and bulimia nervosa. International Journal of Eating Disorders. 1990;9(3):303-9.

16. McNicholas F. Eating psychopathology and its effect on pregnancy, infant growth and development. Irish Journal of Psychological Medicine. 1996;13(02):67-9.

17. Russell G, Treasure J, Eisler I. Mothers with anorexia nervosa who underfeed their children: their recognition and management. Psychol Med. 1998;28(1):93-108.

18. Stein A, Woolley $\mathrm{H}$. The influence of parental eating disorders on young children: implications of recent research for some clinical interventions. Eating Disorders. 1996;4(2):139-46.

19. Collaboration OS. Estimating the reproducibility of psychological science. Science. 2015;349(6251):aac4716.

20. Olsen J, Melbye M, Olsen SF, Sørensen TI, Aaby P, Andersen A-MN, et al. The Danish National Birth Cohort-its background, structure and aim. Scandinavian journal of public health. 2001;29(4):300-7.

21. DNBC. Danish National Birth Cohort 2012 [25 March 2014]. Available from: http://www.ssi.dk/English/RandD/Research\%20areas/Epidemiology/DNBC/For\%20researchers/Data\%20avai lable.aspx.

22. Micali N, Stemann Larsen P, Strandberg-Larsen K, Nybo Andersen AM. Size at birth and preterm birth in women with lifetime eating disorders: a prospective population-based study. BJOG : an international journal of obstetrics and gynaecology. 2015. 
23. Micali N, Simonoff E, Treasure J. Risk of major adverse perinatal outcomes in women with eating disorders. The British Journal of Psychiatry. 2007;190(3):255-9.

24. Zhu JL, Basso O, Obel C, Hvidtjorn D, Olsen J. Infertility, infertility treatment and psychomotor development: the Danish National Birth Cohort. Paediatric and perinatal epidemiology. 2009;23(2):98-106.

25. Goodman R. The Strengths and Difficulties Questionnaire: a research note. J Child Psychol Psychiatry. 1997;38(5):581-6.

26. Niclasen J, Teasdale TW, Andersen A-MN, Skovgaard AM, Elberling H, Obel C. Psychometric properties of the Danish Strength and Difficulties Questionnaire: the SDQ assessed for more than 70,000 raters in four different cohorts. PloS one. 2012;7(2):e32025.

27. Goodman R. Psychometric properties of the strengths and difficulties questionnaire. J Am Acad Child Adolesc Psychiatry. 2001;40(11):1337-45.

28. Angold A, Costello EJ, Erkanli A. Comorbidity. J Child Psychol Psychiatry Allied Discip. 1999;40(1):57-

87.

29. Niclasen J. SDQ Danish cut off scores: 5-7-year-olds [01/03/2014].

30. Mackinnon DP, Dwyer JH. Estimating mediated effects in prevention studies. Evaluation review. 1993;17(2):144-58.

31. Micali N, De Stavola B, Ploubidis GB, Simonoff E, Treasure J. The effects of maternal eating disorders on offspring childhood and early adolescent psychiatric disorders. Int J Eat Disord. 2014;47(4):385-93.

32. Yoshimasu K, Barbaresi WJ, Colligan RC, Voigt RG, Killian JM, Weaver AL, et al. Childhood ADHD is strongly associated with a broad range of psychiatric disorders during adolescence: a population-based birth cohort study. J Child Psychol Psychiatry. 2012;53(10):1036-43.

33. Biederman J, Petty CR, Monuteaux MC, Fried R, Byrne D, Mirto T, et al. Adult psychiatric outcomes of girls with attention deficit hyperactivity disorder: 11-year follow-up in a longitudinal case-control study. American Journal of Psychiatry. 2010;167(4):409-17.

34. Sonneville KR, Calzo JP, Horton NJ, Field AE, Crosby RD, Solmi F, et al. Childhood hyperactivity/inattention and eating disturbances predict binge eating in adolescence. Psychol Med. 2015; FirstView:1-10.

35. Nazar BP, Pinna CMdS, Coutinho G, Segenreich D, Duchesne M, Appolinario JC, et al. Review of literature of attention-deficit/hyperactivity disorder with comorbid eating disorders. Revista Brasileira de Psiquiatria. 2008;30(4):384-9.

36. Seitz J, Kahraman-Lanzerath B, Legenbauer T, Sarrar L, Herpertz S, Salbach-Andrae H, et al. The role of impulsivity, inattention and comorbid ADHD in patients with bulimia nervosa. PLoS One. 2013;8(5):e63891.

37. Reinblatt SP. Are Eating Disorders Related to Attention Deficit/Hyperactivity Disorder? Current treatment options in psychiatry. 2015;2(4):402-12.

38. Wade TD, Bulik CM, Neale M, Kendler KS. Anorexia nervosa and major depression: shared genetic and environmental risk factors. American Journal of Psychiatry. 2000;157(3):469-71.

39. Keel PK, Klump KL, Miller KB, McGue M, lacono WG. Shared transmission of eating disorders and anxiety disorders. International Journal of Eating Disorders. 2005;38(2):99-105.

40. Kothari R, Barona M, Treasure J, Micali N. Social Cognition in Children at Familial High-risk of Developing an Eating Disorder. Under review.

41. Fahy T, Treasure J. Children of mothers with bulimia nervosa. BMJ: British Medical Journal. 1989;299(6706):1031.

42. Crockenberg S, Acredolo C. Infant temperament ratings: A function of infants, of mothers, or both? Infant Behavior and Development. 1983;6(1):61-72.

43. Lopez C, Stahl D, Tchanturia K. Estimated intelligence quotient in anorexia nervosa: a systematic review and meta-analysis of the literature. Annals of General Psychiatry. 2010;9(1):1-10.

44. Micali N, De Stavola B, dos-Santos-Silva I, Steenweg-de Graaff J, Jansen P, Jaddoe V, et al. Perinatal outcomes and gestational weight gain in women with eating disorders: a population-based cohort study. BJOG: An International Journal of Obstetrics \& Gynaecology. 2012;119(12):1493-502. 
45. Keski-Rahkonen A, Sihvola E, Raevuori A, Kaukoranta J, Bulik CM, Hoek HW, et al. Reliability of selfreported eating disorders: Optimizing population screening. International Journal of Eating Disorders. 2006;39(8):754-62. 
Table 1. Socio demographics across sample studied: comparison using chi-square and F-tests depending on variable

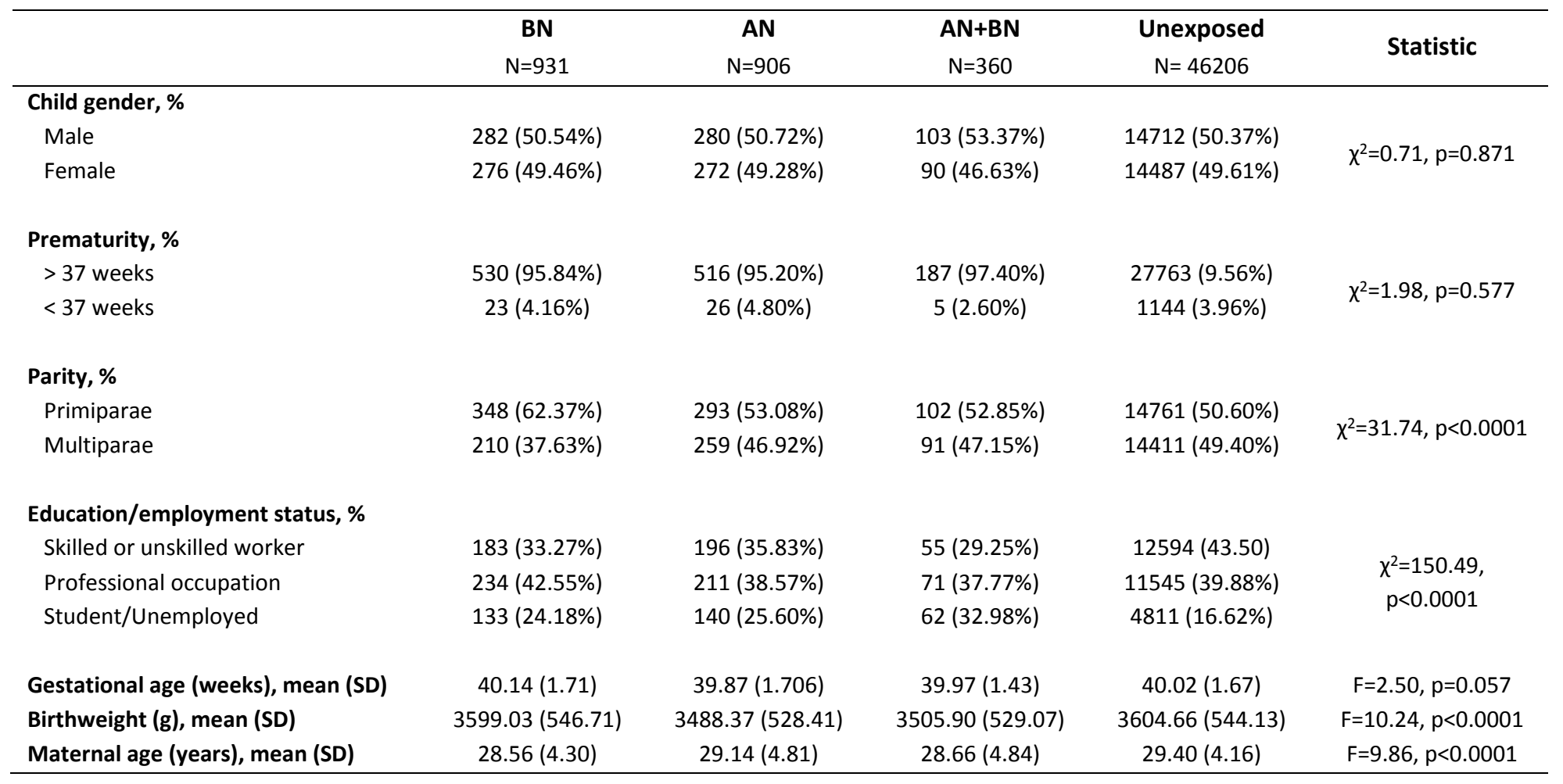


Table 2. Maternal eating disorders and child's developmental, temperament and attachment variables at 18 months: odds ratios and (95\%confidence intervals) from logistic regression

\begin{tabular}{|c|c|c|c|c|c|c|c|c|}
\hline & \multicolumn{4}{|c|}{ Crude OR $(95 \% \mathrm{Cl})$} & \multicolumn{4}{|c|}{ Adjusted ${ }^{\text {a }}$ OR $(95 \% \mathrm{Cl})$} \\
\hline & $\mathrm{BN}(\mathrm{N}=446)$ & AN $(N=460)$ & $A N+B N(N=167)$ & $\begin{array}{l}\text { Unexposed } \\
(\mathrm{N}=23458)\end{array}$ & $B N(N=446)$ & AN $(N=460)$ & $A N+B N(N=167)$ & $\begin{array}{l}\text { Unexposed } \\
(\mathrm{N}=23458)\end{array}$ \\
\hline \multicolumn{9}{|l|}{ Female } \\
\hline Delayed motor development & $1.12(0.63-2.00)$ & $0.58(0.26-1.31)$ & $0.56(0.14-2.26)$ & Ref. & $1.18(0.66-2.12)$ & $0.56(0.25-1.27)$ & $0.54(0.13-2.20)$ & Ref. \\
\hline Del. cog/language development & $0.87(0.72-1.06)$ & $0.86(0.71-1.05)$ & $0.81(0.52-1.04)$ & Ref. & $0.91(0.75-1.11)$ & $0.88(0.72-1.08)$ & $0.76(0.53-1.09)$ & Ref. \\
\hline Difficulties in attachment & $1.96 * * *(1.34-2.85)$ & $1.70 *(1.13-2.56)$ & $1.97 *(1.03-3.76)$ & Ref. & $2.07 * * *(1.42-3.03)$ & $1.69 *(1.12-2.55)$ & $1.96 *(1.02-3.74)$ & Ref. \\
\hline \multirow[t]{2}{*}{ Child's difficult temperament } & $1.91^{* * *}(1.34-2.73)$ & $1.58^{*}(1.06-2.34)$ & $1.15(0.54-2.47)$ & Ref. & $1.85^{* * *}(1.30-2.65)$ & $1.46(0.98-2.19)$ & $1.07(0.50-2.29)$ & Ref. \\
\hline & $\mathrm{BN}(\mathrm{N}=491)$ & AN $(N=449)$ & $A N+B N(N=193)$ & $\begin{array}{l}\text { Unexposed } \\
(\mathrm{N}=22258)\end{array}$ & $B N(N=491)$ & AN (N=449) & $A N+B N(N=193)$ & $\begin{array}{l}\text { Unexposed } \\
(\mathrm{N}=22258)\end{array}$ \\
\hline \multicolumn{9}{|l|}{ Male } \\
\hline Delayed motor development & $0.73(0.41-1.30)$ & $0.73(0.41-1.30)$ & $0.69(0.26-1.88)$ & Ref. & $0.77(0.43-1.38)$ & $0.71(0.40-1.27)$ & $0.77(0.23-1.72)$ & Ref. \\
\hline Del. cog/language development & $0.91(0.76-1.10)$ & $0.73^{* * *}(0.60-0.88)$ & $0.90(0.66-1.23)$ & Ref. & $0.94(0.78-1.13)$ & $0.75^{* *}(0.62-0.91)$ & $0.92(0.67-1.26)$ & Ref. \\
\hline Difficulties in attachment & $1.63 * *(1.15-2.30)$ & $1.44 *(1.00-2.07)$ & $1.14(0.58-2.23)$ & Ref. & $1.71 * *(1.21-2.41)$ & $1.39(0.96-2.02)$ & $1.12(0.57-2.21)$ & Ref. \\
\hline Child's difficult temperament & $1.47+(0.98-2.22)$ & $1.60 * *(1.08-2.38)$ & $2.44 * *(1.40-4.24)$ & Ref. & $1.36(0.89-2.06)$ & $1.56^{*}(1.05-2.31)$ & $2.33^{* *}(1.34-4.07)$ & Ref. \\
\hline
\end{tabular}

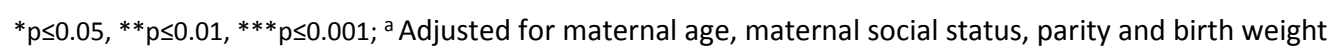


Table 3 Maternal eating disorder and child psychopathology at 7 years of age: Odds ratios and (95\%confidence intervals) from ordinal logistic regression.

\begin{tabular}{|c|c|c|c|c|c|c|c|c|}
\hline & \multicolumn{4}{|c|}{ Crude OR (95\% Cl) } & \multicolumn{4}{|c|}{ Adjusted a OR $(95 \% \mathrm{Cl})$} \\
\hline & $\mathrm{BN}(\mathrm{N}=427)$ & AN $(N=438)$ & $A N+B N(N=161)$ & $\begin{array}{l}\text { Unexposed } \\
(\mathrm{N}=21483)\end{array}$ & $\mathrm{BN}(\mathrm{N}=427)$ & AN $(N=438)$ & $A N+B N(N=161)$ & $\begin{array}{l}\text { Unexposed } \\
(\mathrm{N}=21483)\end{array}$ \\
\hline \multicolumn{9}{|l|}{ Female } \\
\hline Total difficulties & $1.04(0.81-1.32)$ & $1.25(0.99-1.56)$ & $1.35(0.94-1.94)$ & Ref. & $1.00(0.78-1.27)$ & $1.19(0.95-1.50)$ & $1.30(0.90-1.87)$ & Ref. \\
\hline Emotional difficulties & $1.08(0.83-1.40)$ & $1.34 * *(1.05-1.71)$ & $1.44(0.98-2.12)$ & Ref. & $0.99(0.76-1.29)$ & $1.28 *(1.00-1.63)$ & $1.34(0.91-1.99)$ & Ref. \\
\hline Conduct difficulties & $1.39 * *(1.07-1.80)$ & $1.10(0.84-1.45)$ & $0.80(0.48-1.34)$ & Ref. & $1.41 * *(1.09-1.83)$ & $1.08(0.82-1.43)$ & $0.79(0.48-1.32)$ & Ref. \\
\hline Hyperactivity difficulties & $1.23(0.98-1.54)$ & $1.04(0.82-1.31)$ & $1.23(0.85-1.79)$ & Ref. & $1.20(0.96-1.51)$ & $1.00(0.79-1.27)$ & $1.20(0.83-1.74)$ & Ref. \\
\hline Peer difficulties & $1.13(0.89-1.44)$ & $1.17(0.93-1.48)$ & $1.32(0.91-1.91)$ & Ref. & $1.12(0.88-1.43)$ & $1.15(0.91-1.46)$ & $1.29(0.88-1.87)$ & Ref. \\
\hline \multirow[t]{2}{*}{ Pro-social difficulties } & $1.02(0.80-1.31)$ & $1.08(0.85-1.37)$ & $1.03(0.70-1.53)$ & Ref. & $1.05(0.83-1.35)$ & $1.11(0.87-1.40)$ & $1.07(0.72-1.59)$ & Ref. \\
\hline & $\mathrm{BN}(\mathrm{N}=473)$ & $A N(N=432)$ & $A N+B N(N=185)$ & $\begin{array}{l}\text { Unexposed } \\
(\mathrm{N}=22557)\end{array}$ & $\mathrm{BN}(\mathrm{N}=473)$ & AN $(N=432)$ & $\mathrm{AN}+\mathrm{BN}(\mathrm{N}=185)$ & $\begin{array}{l}\text { Unexposed } \\
(\mathrm{N}=22557)\end{array}$ \\
\hline \multicolumn{9}{|l|}{ Male } \\
\hline Total difficulties & $1.58^{* * *(1.29-1.93)}$ & $1.40 * *(1.13-1.74)$ & $1.95^{* * *}(1.44-2.64)$ & Ref. & $1.51 * * *(1.23-1.84)$ & $1.37 * *(1.10-1.70)$ & $1.91 * * *(1.41-2.59)$ & Ref. \\
\hline Emotional difficulties & $1.32 *(1.04-1.68)$ & $1.62 * * *(1.28-2.05)$ & $1.94 * * *(1.39-2.72)$ & Ref. & $1.23(0.97-1.57)$ & $1.56 * * *(1.23-1.98)$ & $1.92 * * *(1.36-2.70)$ & Ref. \\
\hline Conduct difficulties & $1.36 * *(1.08-1.70)$ & $1.32 *(1.04-1.68)$ & $1.32(0.92-1.90)$ & Ref. & $1.32 *(1.05-1.66)$ & $1.32 *(1.04-1.67)$ & $1.28(0.89-1.83)$ & Ref. \\
\hline Hyperactivity difficulties & $1.37 * *(1.11-1.69)$ & $1.01(0.79-1.27)$ & $1.18(0.84-1.67)$ & Ref. & $1.33^{* *(1.08-1.64)}$ & $1.00(.078-1.26)$ & $1.16(0.82-1.64)$ & Ref. \\
\hline Peer difficulties & $1.40 * * *(1.14-1.70)$ & $1.21(0.97-1.50)$ & $1.74 * * *(1.29-2.35)$ & Ref. & $1.37 * *(1.12-1.67)$ & $1.19(0.96-1.48)$ & $1.71 * * *(1.26-2.32)$ & Ref. \\
\hline Pro-social difficulties & $1.11(0.88-1.40)$ & $0.94(0.73-1.22)$ & $1.39(0.99-1.96)$ & Ref. & $1.14(0.91-1.44)$ & $0.94(0.73-1.22)$ & $1.40(0.99-1.97)$ & Ref. \\
\hline
\end{tabular}

${ }^{*} \mathrm{p} \leq 0.05,{ }^{* *} \mathrm{p} \leq 0.01,{ }^{* * *} \mathrm{p} \leq 0.001 ;{ }^{\text {a }}$ Adjusted for maternal age, maternal education/employment, parity and birth weight 